NICOLE PIETRI

\title{
CHRISTIAN BOLTANSKI : UN ARTISTE MINIMALISTE EXPRESSIONNISTE
}

Christian Boltanski est un artiste français né le 6 septembre 1944, quelques jours après la libération de Paris. L'artiste vit et travaille dans son atelier de la banlieue parisienne, à Malakoff. Il est reconnu internationalement et ses œuvres sont présentes dans de prestigieuses collections privées ou dans celles des plus grands musées du monde. Son activité artistique qu'il poursuit encore aujourd'hui couvre près d'un demi-siècle. Vers l'âge de 14 ans, Christian Boltanski a quitté l'école et a commencé à peindre des œuvres figuratives en couleurs, de grands formats, et il a ensuite abandonné ce médium à la fin des années soixante. Les œuvres qu'il produit aujourd'hui ne sont plus des peintures et Christian Boltanski est surtout connu pour ses installations. Cependant, malgré que toute peinture soit absente de sa production plastique, il continue à revendiquer une filiation avec la peinture traditionnelle et, depuis sa première exposition en mai 1968 au cinéma Le Ranelagh, l'artiste se dit « peintre $»^{1}$. Il situe son travail entre minimaliste et expressionnisme. Ce qui semble, au premier abord tout à fait paradoxal, ce n'est pas que Christian Boltanski se présente comme un peintre alors qu'il ne peint plus, c'est qu'il associe l'expressionnisme au minimalisme. En effet, ces deux courants artistiques ne sont-ils pas aux antipodes?

\section{Un art qui hurle?}

En évoquant ses débuts dans la peinture, Christian Boltanski déclare à l'historienne d'art Catherine Grenier avec laquelle il a réalisé un livre d'entretiens en 2007 : " Je pense vraiment que je suis un peintre expressionniste » tout en précisant : « Ce que j'aime, c'est l'art expressionniste, l'art qui hurle » tout en concédant qu'il «n'aurait jamais osé dire ça il y a vingt ans $»^{2}$. En se positionnant du côté de l'art qui hurle, Boltanski semble se référer à l'expressionisme du début du vingtième siècle. Dans l'article que Sylvain Bourmeau lui consacre, Christian Boltanski précise : « Je suis un peintre figuratif, tout à fait figuratif, très traditionnel, un peintre qui travaille sur les émotions » et l'artiste ajoutait : «à quelques différences près, je ne pense pas que mon exposition actuelle soit très différente d'une exposition de tableaux : c'est une sorte de grande fresque

\footnotetext{
${ }^{1}$ Delphine Renard, «Entretien avec Christian Boltanski », in Boltanski, Paris, Centre Georges Pompidou, 1984, p. 85

${ }^{2}$ Christian Boltanski, Catherine Grenier, La vie possible de Christian Boltanski [2007], Paris, Seuil, 2010, p. 99.
} 
expressionniste $»^{3}$. L'œuvre de Christian Boltanski, comme on l'a si souvent noté, n'est-elle pas, comme nombre de celles produites par les expressionnistes, marquée par la mort ? En effet, Christian Boltanski fait ici référence à son exposition intitulée Concessions qui s'est tenue à la galerie parisienne d'Yvon Lambert du 24 février au 13 avril 1996 et dans laquelle on pouvait voir des images de cadavres tirées de la revue à sensation espagnole El Caso. La vue de ces photographies, installées aux murs, n'étaient pas directement accessibles à la vue car elles étaient recouvertes de tissus que de petits ventilateurs soulevaient très légèrement. Christian Boltanski, par les réponses qu'il apporte au travers des propos rapportés par Catherine Grenier et Sylvain Bourmeau, nous donne des éléments clés pour aborder son œuvre, sans faire de la peinture, l'artiste n'était-il pas attaché à l'image et à la dimension figurative, narrative et expressionniste de son œuvre ? Néanmoins, Christian Boltanski confiait à Étienne Hatt qu'il a commencé son activité artistique à la période du minimalisme et que cela avait « refroidi son travail $»^{4}$. De même que, interrogé par Catherine Grenier, Christian Boltanski explique que son « vocabulaire, depuis le début, est principalement minimaliste $»^{5}$. L'artiste se réfère à la boîte à biscuit qui occupe une place importante dans son œuvre : elle est présente dans sa pratique plastique depuis les années soixante-dix et on la retrouve depuis, de facto, dans un très grand nombre d'installations. De plus, l'artiste l'avait lui-même qualifiée d'objet " minimaliste " ${ }^{6}$. Néanmoins, on ne sera pas surpris que Christian Boltanski envisage la boîte à biscuits à la fois sous l'angle du parallélépipède rectangle et à la fois comme une urne funéraire. Du reste, Christian Boltanski, évoquant ses Reconstitutions ${ }^{7}$, dissocie le contenu de ces boîtes (vitrines ou tiroirs) qu'il qualifie d'« ob-jets expressionnistes » du contenant qu'il compare à des «tiroirs de morgue $»^{8}$ et de l'artiste minimaliste Donald Judd.

Ne pourrait-on prêter à Christian Boltanski ces mots du précurseur de l'expressionnisme Edward Munch : « Dès l'instant de ma naissance, les anges de

\footnotetext{
${ }^{3}$ Sylvain Bourmeau, « Christian Boltanski - Le prêche de l'escroc », Les Inrocks, [en ligne], 3 avril 1996, consulté en février 2018, disponible sur http://www.lesinrocks.com/1996/04/03/musique/concerts/christian- boltanski-le-preche-de-lescroc$11236259 /$.

${ }^{4}$ Étienne Hatt, « D'autres vies que la mienne », in Alain Fleischer, Étienne Hatt, Richard Leydier, Michel Nuridsany, Didier Semin, Les grands entretiens d'Artpress : Christian Boltanski, Paris, IMEC éditeur - Artpress, 2014, p. 59.

${ }^{5}$ Christian Boltanski, Catherine Grenier, La vie possible de Christian Boltanski, p. 99.

${ }^{6}$ Elisabeth Lebovici, «Bacon s'est mis à avoir l'air d'un Bacon, moi, d'une boîte de biscuits », Libération, le 1 novembre 2003, 6989, pp. 42, 43.

${ }^{7}$ Dans ses « Reconstitutions », il y a des objets réalisés par l'artiste comme nous l'indique le titre de l'œuvre conservée au Musée d'art moderne de Saint Etienne : Reconstitution, essai de reconstitution en pâte à modeler effectué le 15 novembre 1970 d'un fusil en bois que possédait C.B. entre 1949 et 1951.

${ }^{8}$ Christian Boltanski, Catherine Grenier, La vie possible de Christian Boltanski, p. 99.
} 
l'anxiété, de l'inquiétude et de la mort se tinrent à mes côtés ${ }^{9}$ ? N'a-t-il pas été très marqué par les circonstances qui ont été à l'origine de sa naissance ? Ses parents inquiets de la tournure que prenaient les évènements en France et conscients du danger que cela représentait pour les juifs, avaient simulé une violente dispute pour être entendus de leurs voisins, puis son père avait disparu. Pour accréditer leur histoire et protéger ses biens, sa mère, catholique, avait divorcé. Comme beaucoup de juifs, son père Etienne Boltanski s'était résigné à se cacher ; il vivra ainsi près de deux ans dans un petit espace qui se trouvait entre les étages de l'appartement que la famille Boltanski occupait rue de Grenelle à Paris.

Christian Boltanski a été conçu pendant cette période-là et il a, dès son plus jeune âge, entendu des histoires de survivants racontées par les amis de ses parents qui tous s'étaient cachés ou revenaient des camps. Christian Boltanski raconte qu'il a vécu une enfance pour le moins étrange et fortement anxiogène en raison de ces récits dont on imagine aisément l'émotion qui devait s'en dégager. De plus, ces récits étaient particulièrement présents puisqu'ils constituaient comme nous le dit Christian Boltanski « l'unique sujet de conversation » ${ }^{\mathbf{1 0}}$. L'ambiance dans laquelle Boltanski a baigné durant son enfance ne le conduisait-il pas à être particulièrement proche de l'expressionnisme à la fois par les émotions qu'il entend susciter chez ceux qui abordent son œuvre et par les images qu'il réalise ? La boîte à biscuits, parallélépipède rectangle et élément emblématique de son œuvre, le rapproche-t-elle davantage du minimalisme ? L'expressionnisme ne relève-il pas d'un cri, d'une souffrance qui s'expriment de façon exacerbée, tandis que le minimalisme tient à évacuer toutes formes d'affects et de subjectivité ? Comment l'artiste a-t-il géré ce paradoxe au sien de son œuvre ?

\section{Le cri traverserait-il l'œuvre de Boltanski?}

En ce qui concerne la relation de Christian Boltanski à l'expressionnisme et aux images et plus particulièrement à ses peintures, rappelons que Christian Boltanski racontait à Catherine Grenier que, lorsqu'il peignait, l'un de ses deux sujets de prédilection était le Massacre des Innocents ${ }^{11}$. A l'époque où Boltanski peint Le Massacre des innocents, en 1961, il s'inscrit dans une tradition picturale. Il revisite avec plus ou moins de bonheur, un thème qui a inspiré des artistes à différentes époques et bien avant l'avènement de l'expressionnisme et qui relate un épisode de l'Evangile selon saint Matthieu. Le roi Hérode avait ordonné l'exécution de tous les enfants mâles de moins de deux ans de la région de Bethléem car des Mages lui avaient annoncé la naissance d'un enfant appelé à

\footnotetext{
${ }^{9}$ Reinhold Heller, Munch [1984]. Traduit par Sabine Boulongne, Paris, Flammarion, 1991, p. 10.

${ }^{10}$ Christian Boltanski, Catherine Grenier, La vie possible de Christian Boltanski, p. 21.

${ }^{11}$ Ibidem, pp. 25-26.
} 
devenir le roi des Juifs ${ }^{12}$. Dans sa peinture représentant Le Massacre des Innocents ${ }^{13}$, Nicolas Poussin met au premier plan de son tableau une femme la bouche grande ouverte : la raison qui pousse cette femme à crier est explicite et la scène représentée par le peintre parfaitement lisible. Il s'agit d'une femme qui crie parce qu'un soldat s'apprête à tuer son enfant gisant au sol et déjà blessé. Le titre du tableau nous conforte dans cette interprétation par sa référence à un épisode biblique connu. Malgré tout, cette histoire racontée par le tableau de Poussin est indépendante de son auteur. Elle fait partie d'une culture commune, que nous partageons et qui relève d'une narration et d'une fiction, ce qui n'est pas sans faire écho à la production plastique de Christian Boltanski et à son histoire car il n'a pas directement vécu la Shoah, qui fut l'un des plus grands massacres de ce vingtième siècle.

A propos de la Shoah, l'artiste dira à Catherine Grenier que « c'est sans aucun doute l'évènement principal qui a totalement conditionné ma vie $»^{14}$. Le trop petit nombre des peintures de Boltanski auxquelles nous avons accès ne nous permet pas de nous prononcer sur le caractère expressionniste de son œuvre picturale. Toutefois, on peut noter que la question de la mort, de la mort de l'enfant, est déjà au centre des représentations que Christian Boltanski produira par la suite. Néanmoins, lorsque Boltanski s'entretient avec Catherine Grenier, c'est sur le massacre en tant que tel qu'il insiste en précisant : " j'aimais beaucoup les massacres. Je peignais toujours des scènes d'horreur, de guerre, avec plein de personnages, beaucoup de sang ${ }^{15}$. Ainsi, Christian Boltanski présente à Catherine Grenier, une version dramatisée de sa relation à la peinture en y ajoutant l'horreur et le sang. Toutefois, en affichant son goût pour les massacres, Boltanski nous renvoie aussi du côté d'une représentation figurative remplie de cris. L'artiste abandonnera la peinture mais poursuivra son travail avec des équivalents de ses personnages peints. En effet, Christian Boltanski explique à Marie De Brugerolle qu'il avait « décidé que les personnages devaient sortir du tableau » et qu'il avait « fabriqué des grandes poupées grandeur nature $»^{16}$. On pourrait aussi reconnaître la proximité de l'œuvre de Christian Boltanski et du dramaturge polonais Tadeusz Kantor, notamment au travers de La Classe morte ${ }^{17}$ que Boltanski avait

\footnotetext{
12 La famille de Jésus avait fui en Egypte. Ce thème a été traité de nombreuses fois dans la peinture, comme l'a montré l'exposition Poussin, Le Massacre des Innocents, Salle du Jeu de Paume du Domaine de Chantilly, 11 septembre 2017 au 7 janvier 2018.

13 Nicolas Poussin, Le massacre des Innocents, vers 1628, huile sur toile, 1790 x $1180 \mathrm{~cm}$, Domaine de Chantilly, musée Condé.

${ }^{14}$ Christian Boltanski, Catherine Grenier, La vie possible de Christian Boltanski, p. 21.

${ }^{15}$ Ibidem, pp. 25-26

${ }^{16}$ Marie de Brugerolle, "Christian Boltanski. Entretien », in Hors limites. L'art et la vie, 1952 1994, Paris, Centre Georges Pompidou, 1994, pp. 245-253.

${ }^{17}$ De 1975 et joué à Paris, au Théâtre national de Chaillot le 11 octobre 1977. Toutefois Christian Boltanski situe sa rencontre avec le théâtre de Tadeusz Kantor vers 1983. Christian Boltanski, Catherine Grenier, La vie possible de Christian Boltanski, p. 95.
} 
probablement pu voir quelques années plus tard, en 1977, à Paris. Dans cette œuvre de Kantor, chacun des vieillards qui constituent cette classe portent sur leur dos un mannequin : leur double enfantin mort. Du reste l'artiste ne déclarait-il pas à Catherine Grenier : « Je suis mille fois plus proche de Kantor que d'aucun artiste plasticien, c'est absolument mon univers $»^{18}$. Dans les années qui ont suivi la réalisation de ses œuvres peintes et de la représentation des massacres, Christian Boltanski a produit une série de petits films dans lesquels la dimension expressionniste est plus flagrante et le cri plus présent.

\section{De l’image au film}

Christian Boltanski avait intitulé sa première exposition en mai 1968, au cinéma Le Ranelagh : La Vie impossible de C.B. L'artiste présentait une quinzaine de tableaux et de grandes poupées grandeur nature installées dans d'énormes boîtes sur roulettes (sauf une cachée dans l'escalier) et dans lesquelles on pouvait entrer et voir le film La Vie impossible de Christian Boltanski ${ }^{19}$. Cette exposition rassemblait les éléments qui constituent toute la production plastique de l'artiste, passée et à venir. En effet, reprenant ce qu'il avait déjà dit à Marie De Brugerolle quelques années plus tôt et en le développant, Christian Boltanski expliquera à Catherine Grenier qu'il est passé des " tableaux plein de personnages » puis il a ajouté du relief à ses tableaux et ensuite, quittant les tableaux, aux poupées et aux films ${ }^{20}$. En effet, Dans ses films, Christian Boltanski joue de cette ambiguïté entre le personnage qu'il met en scène, l'acteur ou l'actrice (personne réelle) et le mannequin ou la poupée.

Dans un film qu'il a co-réalisé avec Alain Fleisher en 1969, Christian Boltanski met en scène une femme : Christine Pietri ${ }^{21}$ est assise face à la caméra. Malgré tout, son attitude statique et sa chevelure en désordre, sans doute une perruque, font davantage penser à une poupée. Le cadrage est centré sur la partie supérieure de son corps et on ne dispose pas d'élément pour la situer dans un décor. Au début du film, elle sourit, mais elle passe progressivement du sourire à ce que l'on identifie comme un cri, voire un hurlement. Ce film est silencieux, on n'entend pas un cri. A la façon dont la scène est filmée, on pourrait dire que l'image de cette femme qui hurle, sans que l'on entende le moindre son sortir de sa

\footnotetext{
${ }^{18}$ Ibidem, p. 95.

${ }^{19}$ Premier film de Christian Boltanski daté de 1968 et dans lequel il mettait en scène les poupées qu'il avait fabriquées. (Christian Boltanski, Catherine Grenier, La vie possible de Christian Boltanski, p. 35).

${ }^{20}$ Ibidem.

${ }^{21}$ Christine Pietri sera également l'interprète du film de Christian Boltanski co-réalisé avec Alain Fleischer et intitulé Derrière la porte (1970), film cinématographique $16 \mathrm{~mm}$ noir et blanc, sonore, 2'15", distributeur : Light Cone, Paris, Centre Pompidou - Musée national d'art moderne - Centre de création industrielle.
} 
bouche, est comme une suite de tableaux. Ce film silencieux dure trente-cinq secondes. A l'instar du cri de $\mathrm{Munch}^{22}$, on imagine le son sortir de sa bouche et un cri, qui, en quelque sorte, est aussi le nôtre. De plus, lorsque la caméra recule, on entrevoit une blessure. On ne sait pas si c'est cette blessure qui a provoqué son hurlement ou à l'inverse, si, c'est à cause de cette blessure, qu'elle hurle. En tout cas, le titre du film Comment pouvons-nous le supporter $?^{23}$ nous interroge sur ce que nous voyons et sur ce que l'on a du mal à supporter de voir et en même temps nous insupporte, parce que d'une certaine façon nous fait violence et c'est peut-être pour cette raison que le cri de cette femme se confondrait avec le nôtre. Ce film pourrait rejoindre le désir de Francis Bacon de « peindre le cri plutôt que l'horreur »: la source ni la cause du cri ne sont connues. Christian Boltanski affirme " qu'être artiste, c'est mener une très lente psychanalyse sauvage, essayer de résoudre un problème originel ». Selon Boltanski, l'art ne permet pas de résoudre les problèmes mais de mieux vivre avec. Christian Boltanski cachait-il alors ses propres blessures sous des œuvres qui, peut-être, parlaient pour lui, voire hurlaient à sa place ? Néanmoins, selon ses propres dires, Boltanski n'a pas voulu se laisser aller à devenir réellement un artiste expressionniste, il n'a pas voulu se laisser aller à être uniquement celui qui, selon ses propres mots, " crache et étale ». Pourtant, il réalisera un film durant lequel un homme, assis par terre, au fond d'une petite pièce vide à peine éclairée, tousse jusqu'à cracher du sang pendant trois interminables minutes $^{24}$. Une fois de plus, Christian Boltanski établit lui-même ce parallèle entre ses personnages peints et les poupées qu'il fabriquait : "l'image de l'homme qui tousse »dit-il, " c'est exactement comme une poupée...»" ${ }^{25}$, une poupée avec laquelle l'artiste joue, un jeu d'une violence qui provoque un certain malaise chez le spectateur. Dans ce film, L'homme qui tousse, cette fois-ci le son est très présent et donne une dimension expressive très forte à la scène que nous avons sous les yeux. Quel serait le cri de Christian Boltanski ? Celui que l'on entendrait, le sien ou celui d'un autre ou des autres qui ont crié avant lui ?

De la boîte au minimalisme

\footnotetext{
${ }^{22}$ Entre 1893 et 1917, Munch a réalisé cinq versions de son cri : une tempera sur carton se trouve au musée Munch d'Oslo (83,52 x $66 \mathrm{~cm})$, une peinture à l'huile, tempera et pastel sur carton, à la Galerie nationale d'Oslo $(91 \times$ r 73,5 cm), une troisième version appartient également au musée Munch, tandis que la quatrième appartient et une lithographie au Munch d'Oslo (35,5 x 25,4 cm).

${ }^{23}$ Christian Boltanski, Comment pouvons-nous le supporter ? (1969), film cinématographique 16 mm couleur, silencieux, 35", distributeur : Light Cone, Paris, Centre Pompidou - Musée national d'art moderne - Centre de création industrielle.

${ }^{24}$ Christian Boltanski, L'Homme qui tousse (1969), film $16 \mathrm{~mm}$ couleur, sonore, co-réalisation : Jean-Claude Valesy, Cameraman : Alain Thierry, distributeur : Light Cone, Paris, Centre Pompidou - Musée national d'art moderne - Centre de création industrielle.

${ }^{25}$ Christian Boltanski, Catherine Grenier, La vie possible de Christian Boltanski, p. 35.
} 
L'année 1969 durant laquelle Christian Boltanski réalise ses films, marque aussi le début de l'activité artistique qui va compter pour lui. En effet, comme il le dit à Catherine Grenier, il a « commencé à voir des œuvres contemporaines, à comprendre ce qui se passait en art ». Il raconte qu'il a compris que le minimalisme et l'art conceptuel étaient ce qu'il y avait de plus intéressant à l'époque. De plus, Boltanski ne cache pas sa volonté de se situer dans la continuité de l'Histoire de l'art. Né aux Etats-Unis, au milieu des années soixante, le minimalisme réagissait à la subjectivité de l'Expressionnisme abstrait des années quarante. Cependant l'expressionnisme de Boltanski qui n'avait pas rejeté la figuration, se rapproche davantage de celui que l'on a connu en Allemagne ou en Europe au début du $\mathrm{XX}^{\mathrm{e}}$ siècle, celui dans lequel l'expression des affects était manifeste et violente. Donald Judd, l'un des principaux représentants du minimalisme, est plus âgé que Boltanski et a exposé dans la même galerie que lui, à la galerie Templon à Paris. Comme Boltanski, Judd a débuté son activité artistique par la peinture et comme Boltanski, il abandonnera la peinture. Judd croit que l'art ne devrait pas représenter quoi que ce soit, qu'il doit simplement exister. Néanmoins, une des structures en contreplaqué présentée par Judd à la galerie Templon en 1972 était intitulée Box, boîte. Par la suite Judd ne donnera plus de titre à ces œuvres ou tout du moins les nommera sans titre. Judd ne voudra plus orienter la lecture de ses œuvres vers autre chose que ce qu'il donne à voir. Or, La boîte est un titre qui renvoie à l'idée de contenant et par là, même si elle est vide, à ce qu'elle pourrait contenir. La boîte pourrait devenir la boîte de Judd et être pensée en termes de subjectivité, alors que précisément, Judd cherche à mettre à distance la part de subjectivité de l'artiste. D'ailleurs, si Judd exécute ses premières œuvres lui-même et en collaboration avec son père, dès 1966, il se retirera du processus de leur fabrication en la déléguant à des artisans professionnels. Il n'utilisera quasiment plus que le métal. De la même façon, il retirera tout ce qui pourrait renvoyer à une quelconque signification et ses titres deviendront des sans titres: la boîte usinée gardera la même forme mais ne sera plus une boîte, mais un parallélépipède rectangle creux. La machine a remplacé la main de l'artiste. L'œuvre fabriquée de façon industrielle assure à l'artiste d'éliminer tout trace d'imperfection et le sans titre par sa neutralité, permet d'évacuer toute trace qui pourrait renvoyer au vécu de l'artiste ou à celui du regardeur. Le travail de Donald Judd ne nous donne plus à penser qu'il y a quelque chose à chercher à l'intérieur de la boîte. Ainsi, l'œuvre, débarrassée de tout caractère artisanal et de tout affect, de tout ce qui pourrait renvoyer à une figuration, ne fait référence à rien d'autre qu'à elle-même. Seuls comptent la présence du parallélépipède, sa forme, son poids, le matériau dans lequel elle a été fabriquée, ses couleurs et l'agencement des modules qui la constituent. Il n'y a plus de boîte et le moindre contenu à mettre dedans disparaît : désormais, le creux s'est substitué au vide. En 1972, à l'époque où Judd présentait ce qui s'appelait encore Boîte, Christian Boltanski utilisait une sorte d'équivalent des boîtes avec 
ses Vitrines de référence ${ }^{26}$ dans lesquelles il archivait et recyclait des éléments de ses œuvres antérieures constitués d'archives et de la mémoire de sa vie qu'il veut préserver en la mettant sous vitrine. L'historienne d'art américaine Lynn Gumpert, qui est l'auteur de la première monographie sur Christian Boltanski, en 1992, a analysé son recours à la vitrine comme une façon de se "protéger du contenu parfois éprouvant de ses premières œuvres » mais aussi comme une façon de tenir ainsi « son propre travail à distance $»^{27}$.

Boltanski reconnaît par ailleurs qu'il a « un tempérament sans doute trop expressionniste $»^{28}$. D'ailleurs, de son propre aveu, le minimalisme l'a amené à gommer le plus possible tout ce qui était expressionniste en lui, et plus particulièrement la dimension violente, brutale de l'expressionnisme, le plus possible, mais pas tout. Du reste, ce n'était pas son projet puisqu'il n'a pas voulu évacuer la dimension expressionniste de son travail qui touche aux affects. Boltanski a en quelque sorte seulement minoré la part expressionniste de son travail. Boltanski, comme Judd, va intégrer la boîte dans son vocabulaire plastique et privilégier son utilisation car la boîte présente la particularité d'être à la fois un objet minimaliste et expressionniste. Lorsque Boltanski fait référence à Donald Judd, il évoque une des ses œuvres qui, de son point de vue, lui rappelle le travail de Judd. Il s'agit de celle intitulée Trois tiroirs ${ }^{29}$. Boltanski rapproche cette œuvre de celui de Judd pour dire que ces tiroirs qu'il a utilisés sont comme des tiroirs de Morgue et ce qu'il retient alors du minimalisme de Judd, c'est que : " c'est extrêmement froid, métallique ». Il souligne les propriétés physiques des matériaux qui sont directement liées à l'interprétation que l'on peut faire de son travail et du sens qu'il lui donne. Car la sensation physique à laquelle nous renvoie le matériau est importante pour Boltanski, comme pour Judd. Le métal n'est pas, à l'instar du bois, utilisé dans les premières œuvres de Judd, un matériau qui engage au toucher, il est froid et maintient à distance par sa froideur. Du reste, la dimension visuelle des œuvres de Judd prime sur tous les autres sens. Boltanski est attiré par la froideur du matériau mais c'est aussi la présence humaine, la chaleur, la chaleur humaine dont parle Boltanski, c'est la chaleur de la présence humaine que l'artiste va s'appliquer à injecter dans la plupart de ses œuvres. Mais en même temps, la boîte en tant qu'objet minimaliste répond au désir de Boltanski comme il le dit lui-

\footnotetext{
${ }^{26}$ Christian Boltanski, Vitrine de référence, 1971, bois, Plexiglas, photos, cheveux, tissus, papier, terre fil de fer, 12 x $120 \times 59,5 \mathrm{~cm}$, boîte en bois peinte sous plexiglas et contenant 15 éléments de la vie de l'artiste : photos noir et blanc, cheveux, bribes de vêtements de l'artiste, échantillon de son écriture, page de son livre de lecture, entassement de 14 boulettes de terre, un piège composé de trois objets faits de morceaux de tissu, fil de fer, épingles, Paris, Centre Pompidou - Musée national d'art moderne - Centre de création industrielle.

${ }^{27}$ Lynn Gumpert, Christian Boltanski, Paris, Flammarion, 1992, p. 30.

${ }^{28}$ Étienne Hatt, D'autres vies que la mienne, p. 59.

${ }^{29}$ Christian Boltanski, Trois tiroirs, 1970-1971, $44 \mathrm{~cm}$ x 60,5 cm x 40,5 cm, Musée national d'art moderne, Centre Pompidou.
} 
même de « refroidir son art ». On pourrait dire de la boîte qu'elle contient, au sens où elle réprime l'expressionnisme de Boltanski, elle en réprime le cri. Mais, elle reste malgré tout un contenant et elle est, avant tout un contenant qui permet à Boltanski d'apporter à ce minimalisme ce qu'il appelle une " une charge affective ». Les Trois tiroirs étaient fermés par un grillage et chaque tiroir portait une étiquette qui donnait des indications sur ce qui se trouvait à l'intérieur : des petits objets en pâte à modeler ou en plastiline qui reproduisaient ce qui auraient appartenu à Boltanski enfant. L'artiste range ces objets du côté de l'expressionnisme : il les a fabriqués, ils le ramènent à son enfance et ils sont insignifiants mais chargés d'affects car Boltanski associe le bricolage à l'émotion qu'il veut provoquer chez le spectateur « je pense que l'émotion vient d'un côté un peu bricolé, mal fait $»^{30}$. De plus, ils sont fragiles et le fait de les protéger avec le grillage accentue le sentiment que l'on peut avoir de leur fragilité. Les tiroirs comme les vitrines ou les boîtes utilisés par Boltanski ont vocation à contenir quelque chose. La main de l'artiste est, comme pour Judd, exclue du processus de fabrication des tiroirs ou des boîtes, à la différence près que Judd a réalisé les dessins de ses œuvres tandis que la boîte qui va devenir un élément emblématique de Boltanski, on pourrait presque dire sa marque de fabrique, existe déjà dans une production industrielle et n'a pas été réalisée à la demande de l'artiste. Dans l'une de ses premières expositions à la Galerie Templon en 1971, on va voir des boîtes que Boltanski a disposées en piles contre les murs de la Galerie.

Par conséquent, on peut, du point de vue formel, rapprocher ce travail de celui de l'artiste américain Donald Judd. La boîte est interchangeable on peut la remplacer par une autre car rien ne distingue une boîte d'une autre. Elle est solide et faite pour durer alors que dans les objets fabriqués de la main de Boltanski, ceux qui sont à l'intérieur de la boîte, il y a un refus de la perfection et ils sont sans doute voués à disparaître et à emporter avec eux, son vécu, ses souvenirs, sa vie. L'œuvre intitulée Deux boîtes contient un objet long en mie de pain posé sur un carré de tissu blanc duquel sortent des aiguilles, on ne sait pas pendant combien de temps son intégrité sera préservée.

Dans un deuxième temps, Boltanski montrera des boîtes rouillées par ses soins. La répétition et l'empilement des boîtes constituent un tout qui n'effacera pas la singularité de chacune des boites, comme on peut le voir dans le mur de boîte de l'installation Personnes ${ }^{31}$ au Grand Palais ou pour les sortes de tours de boîtes des Réserve des suisses morts ${ }^{32}$ ou encore pour les archives de Christian

\footnotetext{
${ }^{30}$ Christian Boltanski, Catherine Grenier, La vie possible de Christian Boltanski, p. 259.

${ }^{31}$ Christian Boltanski a exposé cette installation durant la troisième édition de la Monumenta qui s'est tenue au Grand palais à Paris du 13 janvier au 21 février 2010.

32 Archives des Suisses morts, 1990, 480 boîtes en métal, photographies et 15 lampes électriques, Musée d'art moderne et contemporain de Saint-Étienne Métropole. Cette installation fait partie d'une série de travaux réalisés par l'artiste entre 1990 et 1991, et présenté à Lausanne cette même année lors de l'exposition Les Suisses morts.
} 
Boltanski, La vie impossible de C.B. ${ }^{33}$. Ces boîtes rouillées sont en quelque sorte affectées par le temps. L'utilisation de la rouille ne serait-elle pas une façon de réintégrer une part d'affect dans les boîtes ? Cette part d'affect tenue à distance par le minimalisme ? La rouille qui agresse ses boîtes n'agirait-elle pas comme une blessure ? Mais Boltanski tient à ce que cette forme de violence faite à la boîte ne dépasse pas un certain seuil. L'atteinte de la matière par la rouille ne doit pas être excessive. Il regrette d'ailleurs que la corrosion des boîtes de l'installation du Grand Hornu en Belgique ${ }^{34}$ soit, de son point de vue, trop importante. Ce n'est pas lui qui s'en est occupé et elles ont été, de son point de vue, trop rouillées.

En 2010, pour l'installation de la Monumenta dans la nef du Grand Palais à Paris, Boltanski fera du froid une composante de son œuvre en demandant que la Monumenta se tienne en hiver. Il jouera de ce froid pour augmenter l'impact physique et émotif de son installation sur les visiteurs. Boltanski, contrairement à Judd, orientera l'interprétation que nous pourrions faire de son œuvre en lui donnant pour titre Personnes (au pluriel) des personnes que l'on assimile à tous ceux de la Shoah, à tous ceux qui ne sont plus : il n'y a plus personne. Alors, dans le froid du Grand Palais et dans son expressionnisme refroidi par le minimalisme, Boltanski nous dira ce qu'il n'arrivait peut-être pas à dire autrement que dans un expressionnisme qui hurle et qui nous affectait, au point de nous demander, à l'image de son film intitulé Comment pouvons-nous le supporter? si ce n'est en refusant d'entendre ce hurlement. Boltanski, en refroidissant son art avec le minimalisme tout en conservant une part d'affect, en réalisant une œuvre emplie d'affect mais dont le cri resterait comme en creux, ne nous permettrait-il pas de le supporter et en supportant ce cri, peut-être, enfin, de le faire entendre ?

\section{BIBLIOGRAPHIE}

BOLTANSKI, Christian, GRENIER, Catherine, La vie possible de Christian Boltanski [2007], Paris, Seuil, 2010.

BOURMEAU, Sylvain, "Christian Boltanski - Le prêche de l'escroc », Les Inrocks, [en ligne], 3 avril 1996, consulté en février 2018, disponible sur http://www.lesinrocks.com/1996/04/03/musique/concerts/christian-boltanski-le-preche-delescroc-11236259/.

BRUGEROLLE, Marie de, «Christian Boltanski. Entretien », in Hors limites. L'art et la vie, 19521994, Paris, Centre Georges Pompidou, 1994, pp. 245-253.

\footnotetext{
33 Christian Boltanski, La vie impossible de C.B., 2001, Bois, grillage métallique, lampe fluorescente, fil électrique, papier, photo, $150 \times 87 \times 12 \mathrm{~cm}, 10 / 15$, ensemble de 20 vitrines accrochées au mur, Centre Pompidou - Musée national d'art moderne - Centre de création industrielle.

${ }^{34}$ Les Registres du Grand-Hornu est la première pièce produite et acquise pour la collection du Musée des Arts Contemporains de la Fédération Wallonie-Bruxelles au Grand-Hornu (MAC's) en 1997, installation, fer blanc, étiquettes, photographies noir \& blanc, lampes de bureau, $472 \times 4015 \times 19 \mathrm{~cm}$.
} 
GUMPERT, Lynn, Christian Boltanski. Traduit par Anne Rochette, Paris, Flammarion, 1992.

HATT, Étienne, " D'autres vies que la mienne », in Les grands entretiens d'Artpress : Christian Boltanski, Paris, IMEC éditeur - Artpress, 2014.

LEBOVICI, Elisabeth, "Bacon s'est mis à avoir l'air d'un Bacon, moi, d'une boîte de biscuits ", Libération, 1 novembre 2003, 6989, pp. 42-43.

REINHOLD, Heller, Munch [1984]. Traduit par Sabine Boulongne, Paris, Flammarion, 1991.

RENARD, Delphine « Entretien avec Christian Boltanski », in Boltanski, Paris, Centre Georges Pompidou, 1984, pp. 70-85.

\title{
CHRISTIAN BOLTANSKI: \\ A MINIMALIST EXPRESSIONIST ARTIST (Abstract)
}

\begin{abstract}
Christian Boltanski is an internationally renowned French artist. His work lies somewhere between two antinomic artistic trends, minimalism and expressionism. On the one hand, minimalism tends to remove all forms of emotions and subjectivity, on the other hand, expressionism expresses a cry, a suffering that would express itself in Boltanski's work. Christian Boltanski was born right after the end of the second world war. He did not directly experience one of the most shocking massacres of the twentieth century, but he grew up listening to the stories of his parent's friends who went into hiding, like his father, or managed to return from the camps. Christian Boltanski's childhood was deeply affected by the stories of those who had survived the Shoah, and the paintings he made at the beginning of his artistic activity represented massacres. Nevertheless, the expressionist dimension of Boltanski's work appeared the most when Boltanski started to explore cinematic art with small movies entitled "The Man who coughs" or "How can we cope with him?". Then, at the end of the 60 s, Boltanski moved away from expressionism and turned to an object with geometric shapes industrially manufactured. The biscuit box, the showcase or the drawer, is given a central place in his work. The artist approached this object with minimalist forms from the angle of a rectangular parallelepiped. However, the box also represents a funeral urn whose function is to preserve the traces of a human existence. Thus, the box full of emotions responds to the artist's desire to transmit emotions to the spectators, while allowing him to contain and erase as much as possible the expressionist aspect of his work. This way, Boltanski's work appears to be the work of both a minimalist and expressionist artist.
\end{abstract}

Keywords: Christian Boltanski, minimalisme, expressionnisme, Shoah, emotions.

\section{CHRISTIAN BOLTANSKI : UN ARTIST MINIMALIST EXPRESIONIST (Rezumat)}

Christian Boltanski este un artist francez de renume mondial. Opera sa se plasează între două tendinţe artistice antinomice, minimalismul şi expresionismul. Pe de o parte, minimalismul tinde să reprime toate formele de afecte şi de subiectivitate, iar pe de altă parte, expresionismul exprimă un strigăt, o suferinţă care transpare în opera lui Boltanski. Christian Boltanski s-a născut la scurt timp după sfârşitul celui de-al doilea război mondial. Nu a trăit în mod direct ceea ce a constituit unul dintre cele mai şocante masacre ale secolului al XX-lea, dar a crescut ascultând poveştile părinţilor prietenilor săi, care fie s-au ascuns, la fel ca tatăl său, fie au reuşit să se întoarcă din lagăre. 
Copilăria lui Christian Boltanski a fost profund afectată de poveştile supravieţuitorilor, iar picturile de la începutul activităţii sale artistice reprezintă masacre. $\mathrm{Cu}$ toate acestea, dimensiunea expresionistă a operei sale a devenit cel mai evidentă prin explorarea artei cinematografice, începând cu filme mici, intitulate „Omul care tuşeşte” sau „Cum îi putem face faţă?”. Apoi, la sfârşitul anilor 1960, Boltanski s-a îndepărtat de expresionism şi s-a orientat către un obiect cu dimensiuni geometrice fabricat pe cale industrială. Cutia de biscuiţi, vitrina sau sertarul dobândesc un loc central în opera sa. Artistul abordează obiectul prin forme minimaliste, din unghiul unui paralelipiped rectangular. În acelaşi timp, cutia poate reprezenta şi o urnă funerară cu funcția de a păstra urmele existenţei umane. Astfel, cutia plină cu emoţii răspunde dorinţei artistului de a transmite emoții spectatorilor, îngăduindu-i să cuprindă sau să șteargă cât de mult posibil aspectul expresionist al lucrării. Prin urmare, lucrările lui Boltanski par a fi opera unui artist deopotrivă minimalist şi expresionist.

Cuvinte-cheie: Christian Boltanski, minimalism, expresionism, Shoah, emoții. 